\title{
Guided Wave Travel Time Tomography for Bends
}

\author{
Arno VOLKER ${ }^{1}$ and Tim van $\mathrm{ZON}^{1}$ \\ ${ }^{1}$ TNO, Stieltjes weg 1, 2600 AD, Delft, The Netherlands \\ Phone: +31 (0)88 86 66292, e-mail: arno.volker@tno.nl
}

\begin{abstract}
.
The concept of predictive maintenance using permanent sensors that monitor the integrity of an installation is an interesting addition to the current method of periodic inspections.

Guided wave tomography has been developed to map the wall thickness using the travel times of guided waves. The method has been demonstrated for straight pipes. The extension of this method to bends is not straightforward because natural focusing occurs due to geometrical path differences. This yields a phase jump, which complicates travel time picking. Because ray-tracing is no longer sufficient to predict the travel times a recursive wave field extrapolation has been developed. The method uses a short spatial convolution operator to propagate a wave field through a bend. The method allows to calculate the wave field at the detector ring, including the phase jump as a consequence of the natural focusing. The recursive wave field extrapolation is done in the space-frequency domain. Therefore dispersion effects can be included easily in the forward modeling. Comparison with measurements shows the accuracy of the method. The tomographic reconstruction is based on the wave field extrapolation kernel.
\end{abstract}

Keywords: Ultrasonic, Tomography, Inversion, Guided wave, Corrosion monitoring, Travel time, Dispersion.

\section{Introduction}

Corrosion is one of the most important mechanisms of structural damage. Periodic inspections are carried out to deal with corrosion risks. But in many cases they are not a cost effective solution. More promising in terms of cost are permanent monitoring systems that can measure the integrity of the installation at any moment in time. The ideal permanent monitoring system for corrosion management should have the following properties. First of all, the coverage of the system should be $100 \%$ of the inside as well as the outside surface of the object. It should also be able to measure the corrosion rate, in order for optimal replacement or repair planning. Finally, the system should be able to work without interference with the production process.

Bends are frequently appearing components in a piping system and are more sensitive to corrosion and erosion than straight pipe segments. This paper will extend our approach to guided wave tomography to bends 


\section{Guided wave propagation through a bend}

To extend our guided wave travel time tomography[1] to bends, we have to understand the wave propagation through bends. The most important complication compared to guided wave propagation in straight pipes is the geometrical focusing that occurs on the knee of the bend. Finite difference modeling[2] is used to study this effect. Some snap shots of the wave propagation are shown in

Figure 1. A point source, which applies an axial force is used to excite guided waves. The center frequency is around $80 \mathrm{kHz}$, such that the wave mode of interest $(\mathrm{L}(0,2)$, the equivalent of the S0-mode in a plate) is hardly dispersive. For geometrical reasons, this wave focuses on the knee of the bend (see

Figure 1b). The wave through the inner part of the bend is the first to arrive at the receiver ring, located on the other side of the bend as can be seen in figure 1c and 1d.

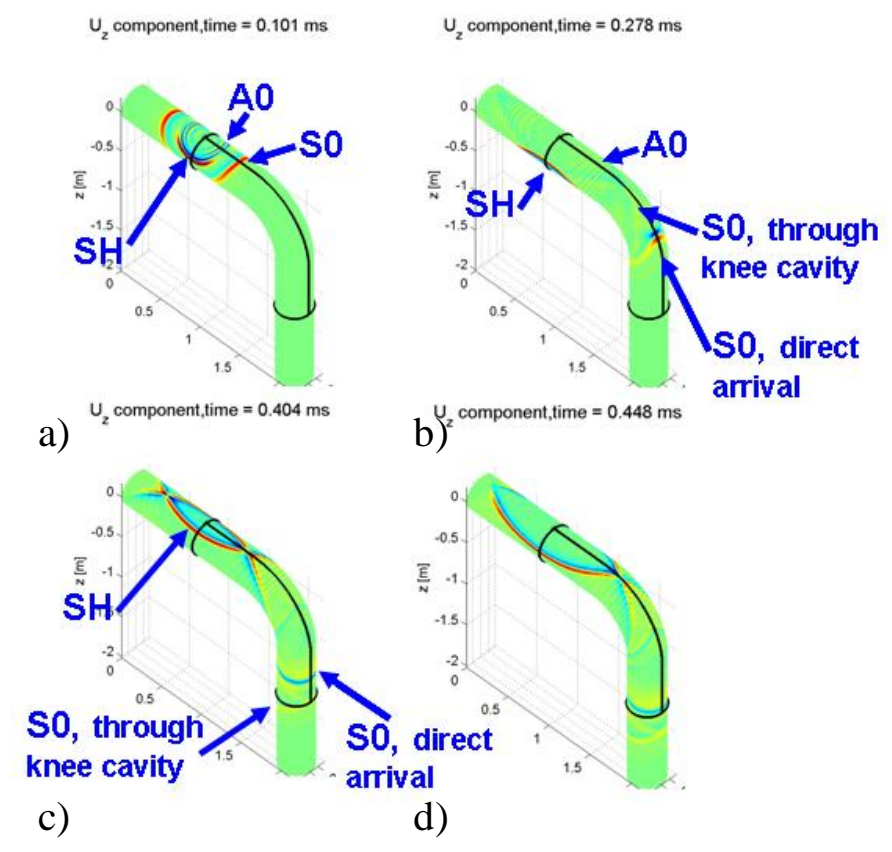

Figure 1 Snap shots of finite difference simulations showing the wave propagation through a bended pipe

The recorded signal is shown Figure 2. The receiver record is copied a few times next to each other, to unwrap the circumferential passes of the wave. The dotted line indicates the calculated travel time using ray-tracing. Looking at the data it immediately becomes apparent that the signals, i.e., the direct waves compared to the ones that went through the focus point have a different phase. For travel time tomography it is important to consistently pick a peak or a trough of the recorded signal. The phase jump, which occurs due to the focus, complicates travel time picking greatly. This requires a forward modeling kernel that includes the phase information, which is difficult to include in our ray-tracing implementation. 


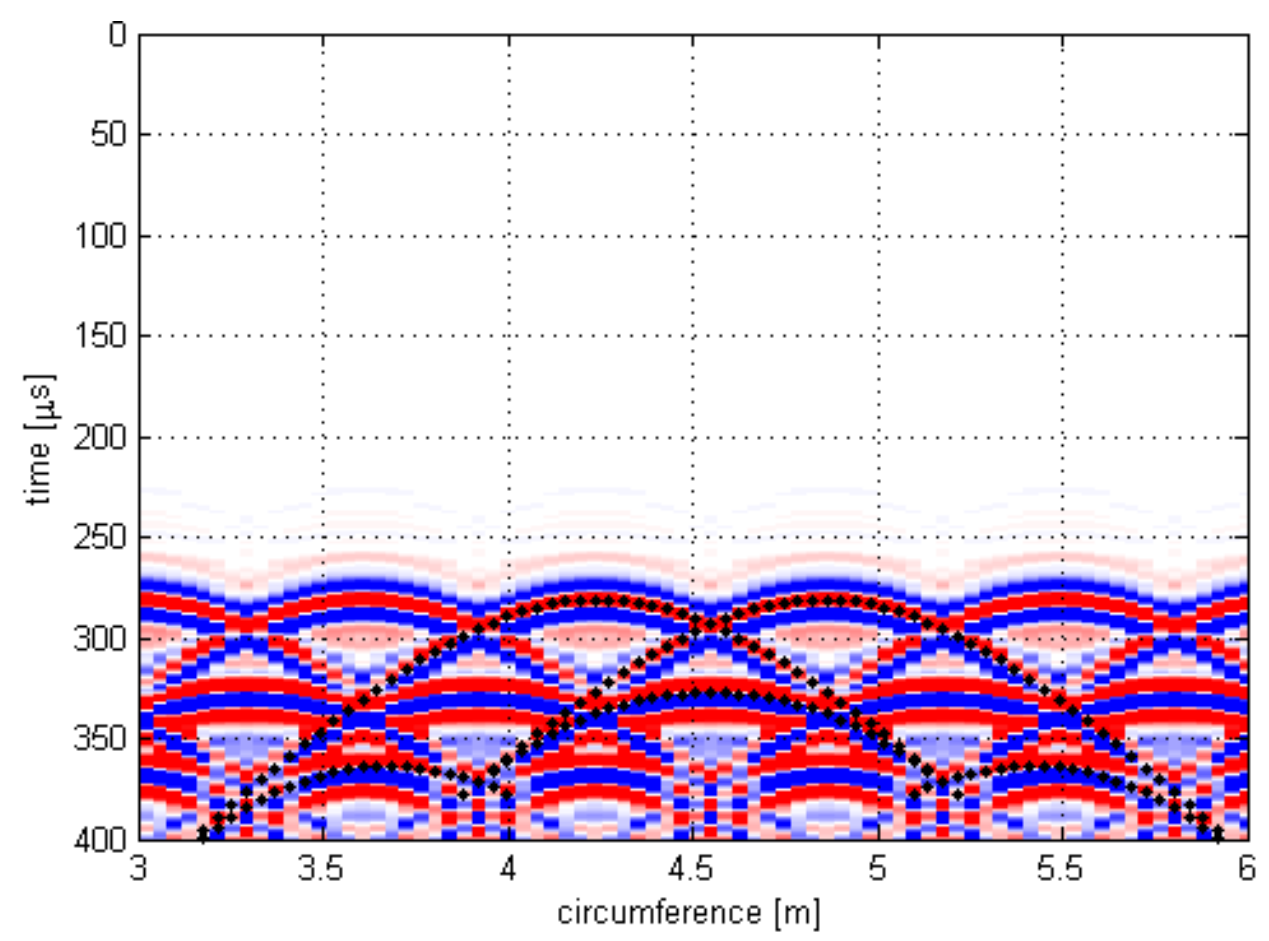

Figure 2 Simulated wave field after propagation through a bend. The recorded field is unwrapped (by copying the recorded field a few times next to each other) and the calculated travel times using ray-tracing are plotted on top of the data (dotted line).

\section{Recrusive wave field extrapolation}

A method that allows us to compute the wave field, including its phase is wave field extrapolation. It is known that a wave field can be extrapolated using a complex exponential in the wave number- frequency domain:

$\exp \left( \pm_{j k} \Delta_{z}\right.$ )

where $\mathrm{kz}$ is the wave number in the propagation direction and $\Delta \mathrm{z}$ is the extrapolation distance. The sign of the argument in the complex exponential determines the propagation direction, either away from the source (forward extrapolation) or towards the source (inverse extrapolation).

This formulation in the wave number-frequency domain means that we cannot deal with lateral velocity variations, which is the result of local wall thickness variations. The solution is that the propagation operator can be applied in the space-frequency domain as a (short) spatial convolution operator.

Taking the inverse Fourier transform of the propagation operator yields in principle an infinitely long operator because it includes angles up to $\pm 90^{\circ}$. To accommodate spatial velocity changes, this operator needs to be short in order to produce accurate results.

A special procedure[3] is used that produces a short convolution operator in a limited angle range. A simple truncation of the operator in the spatial would lead to inaccuracies of the operator in the wavenumber domain. By the recursive approach even small errors 
would lead to incorrect results after many iterations. To reduce the errors, the truncated operator is optimized in the wavenumber domain by using a least-squares estimator. This estimator minimizes the differences between the ideal, long operator and inaccurate, short operator. A weighting matrix is applied to assure a stable operator in the propagating region.

To apply this convolution operator to the bend geometry, the bend is unfolded to 2D plane (see figure 3). The wave field is then extrapolated from one vertical slice to the next one. In the bend, the horizontal extrapolation distance changes along the circumference. The difference in distance will generate the focusing on the outer knee of the bend. To accommodate higher order events, the grid is again copied in the circumferential direction.
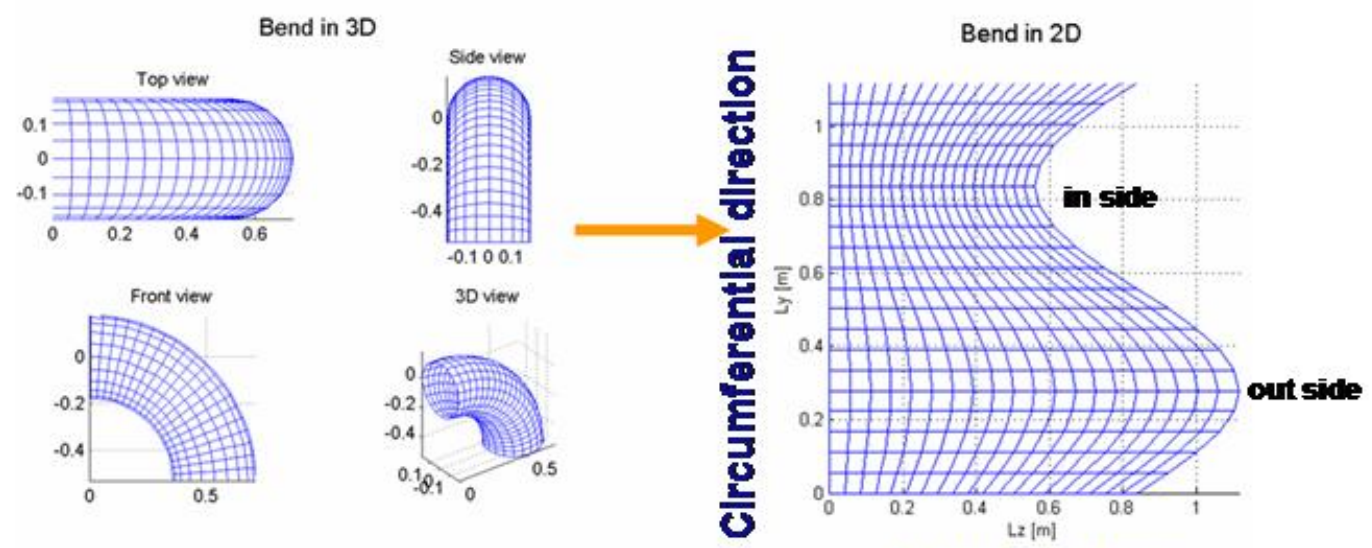

Axial direction

Figure 3 Visualization of 2D Mesh of pipe

The pipe has a nominal wall thickness of $8.3 \mathrm{~mm}$, corresponding to the average wall thickness of the real pipe. The pipe itself has an outer diameter of 14", while the bend radius is $525 \mathrm{~mm}$ and bend angle is $90^{\circ}$. Two straight segments of approximately $1 \mathrm{~m}$ are welded to the ends of the bend. Source and receiver ring are placed at $0.5 \mathrm{~m}$ from the ends of the bend. A forward modeled signal using recursive wave field extrapolation is shown in figure 4. The source is at a similar location as shown in figure 1, i.e., near top of the pipe. The response consists of three branches, the first two arriving branches are the waves that travel through the inner part of the bend. The third branch arrives significantly later than the first two. These are the waves that have traveled through the focus point. Comparing the wavelet phase with the first two arriving branches of the wave field shows indeed a phase difference. Comparison with Figure 2 shows that the wavelet phases are the same as the finite difference modeled data.

Due to limitations in the propagation angle of the extrapolation operator the recorded field does not extend further to higher order of circumferential passes. In the response shown in figure 4 in total 3 circumferential passes were modeled. A pulse with a bandwidth of $130 \%$ around a centre frequency of $150 \mathrm{kHz}$ was used. This is similar to the signal used in actual measurements. 


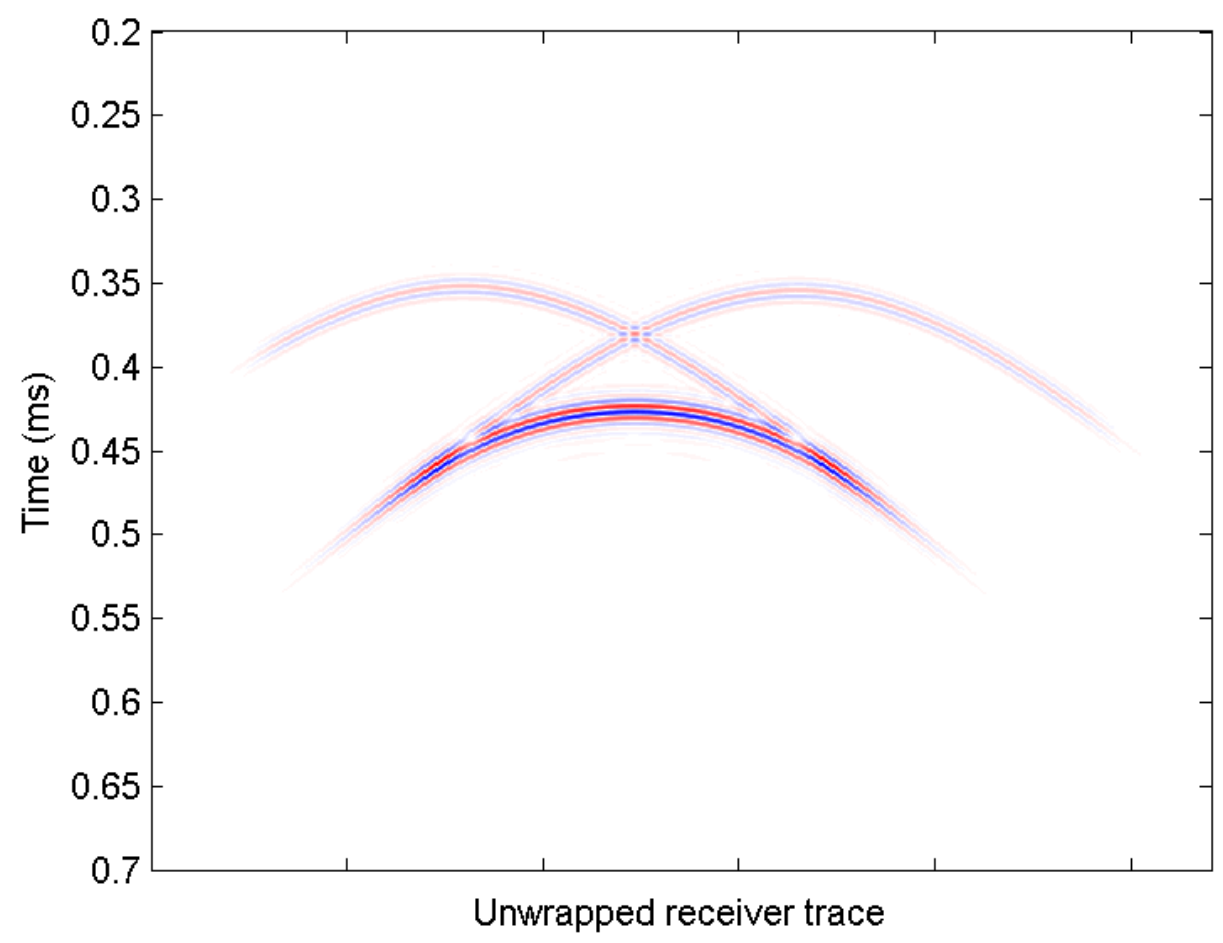

Figure 4. Modeled wave field on the receiver array using recursive wave field extrapolation. The data is shown in an unwrapped view, where higher order helical passes are shown next to each other.

The wave field as shown in Figure 4 is an unwrapped view, i.e., higher order helical passes are shown next to each other. Wrapping the data, by summing all signals that physically arrive at the same receiver produces a very complex interfering wave field. The left and right branches interfere significantly. Since the source is not exactly located at the twelve-o'clock position, there are small timing differences. This clearly shows the difficulties for using travel time based tomography, i.e., significant amount of interference between recorded signals as can be seen in figure 5 . 


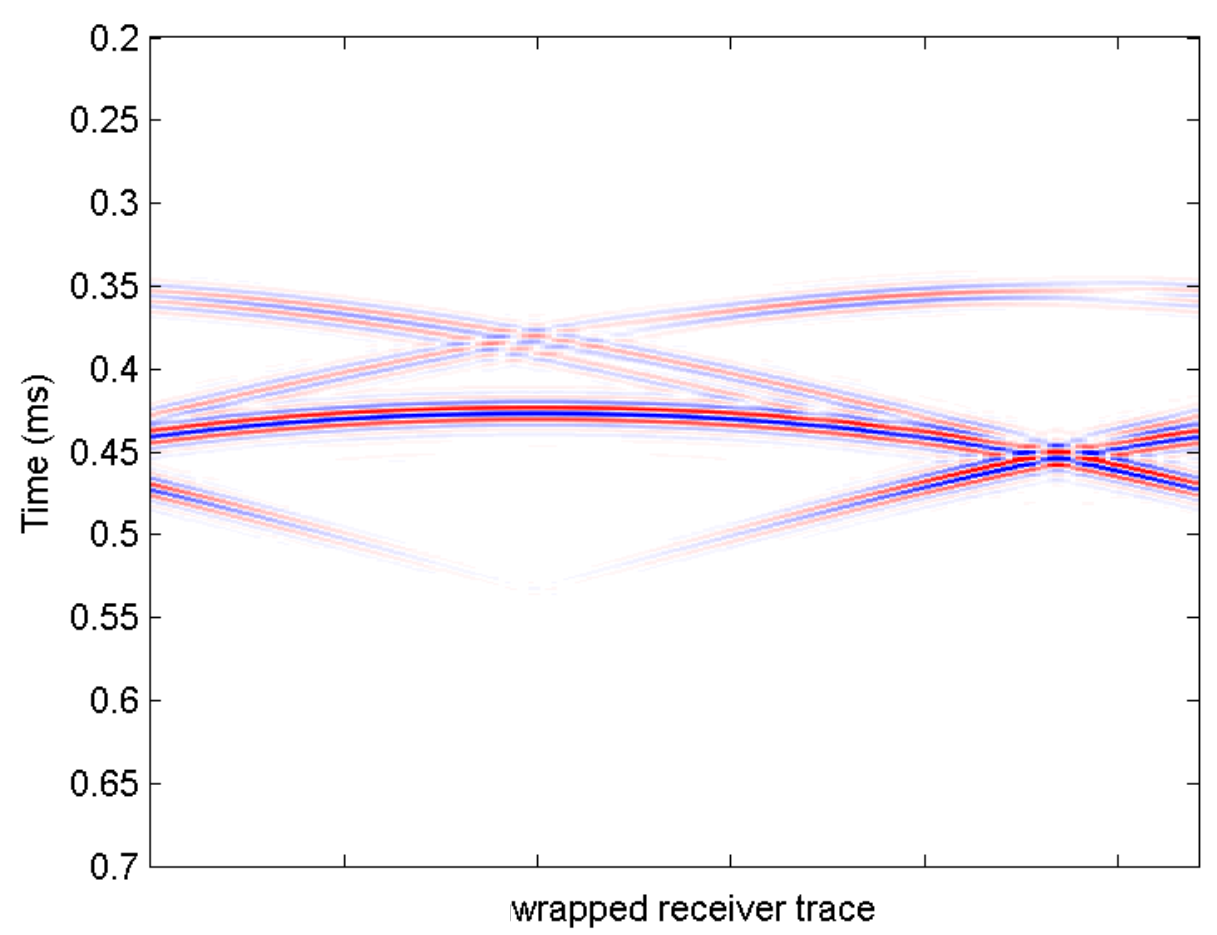

Figure 5 Modeled wave field on the receiver array using recursive wave field extrapolation. The data is shown in a wrapped view, where higher order helical passes are shown at their receiver location. Therefore multiple signals are recorded at the same receiver location.

\section{Comparison to experimental data}

In order to validate our forward modeling, measurements are compared to modeling results on bend. The experimental pipe has the same geometrical properties as the modeled pipe. However, it appeared that the experimental pipe had significant wall thickness variations $( \pm 0.3 \mathrm{~mm})$, especially in the bend sections. For the modeled data the average wall thickness is used. Here the source signal was a linear sweep of $500 \mu \mathrm{s}$ between 50 and $350 \mathrm{kHz}$. After recording the measured data is correlated with the sweep to strongly increase the signal to noise ratio. After this, the signals are corrected for dispersion. Finally, the measured signals are compared to the modeled signals, to evaluate the performance of the kernel based on wave field extrapolation.

This comparison can be seen in figure 6 which shows for four source numbers $(10,15,20$ and 25) the modeled wave field using recursive wave field extrapolation (right side of image) and measured wave field (left side of image). As can be seen the two wave fields match very well with each other for the various sources. First of all, all primary measured events (ignoring dispersion correction residues) are also modeled, even with limited number of circumferential modes taken into account. Also, the travel times match very well. Also the phase shift, which limits travel time tomography, is correctly modeled. There are amplitude differences observed, but they are very likely to be caused by 
directivity patterns of the transducers. The first arrivals are paths that are transmitted at high azimuth angles. These paths propagate through the inner part of the bend.
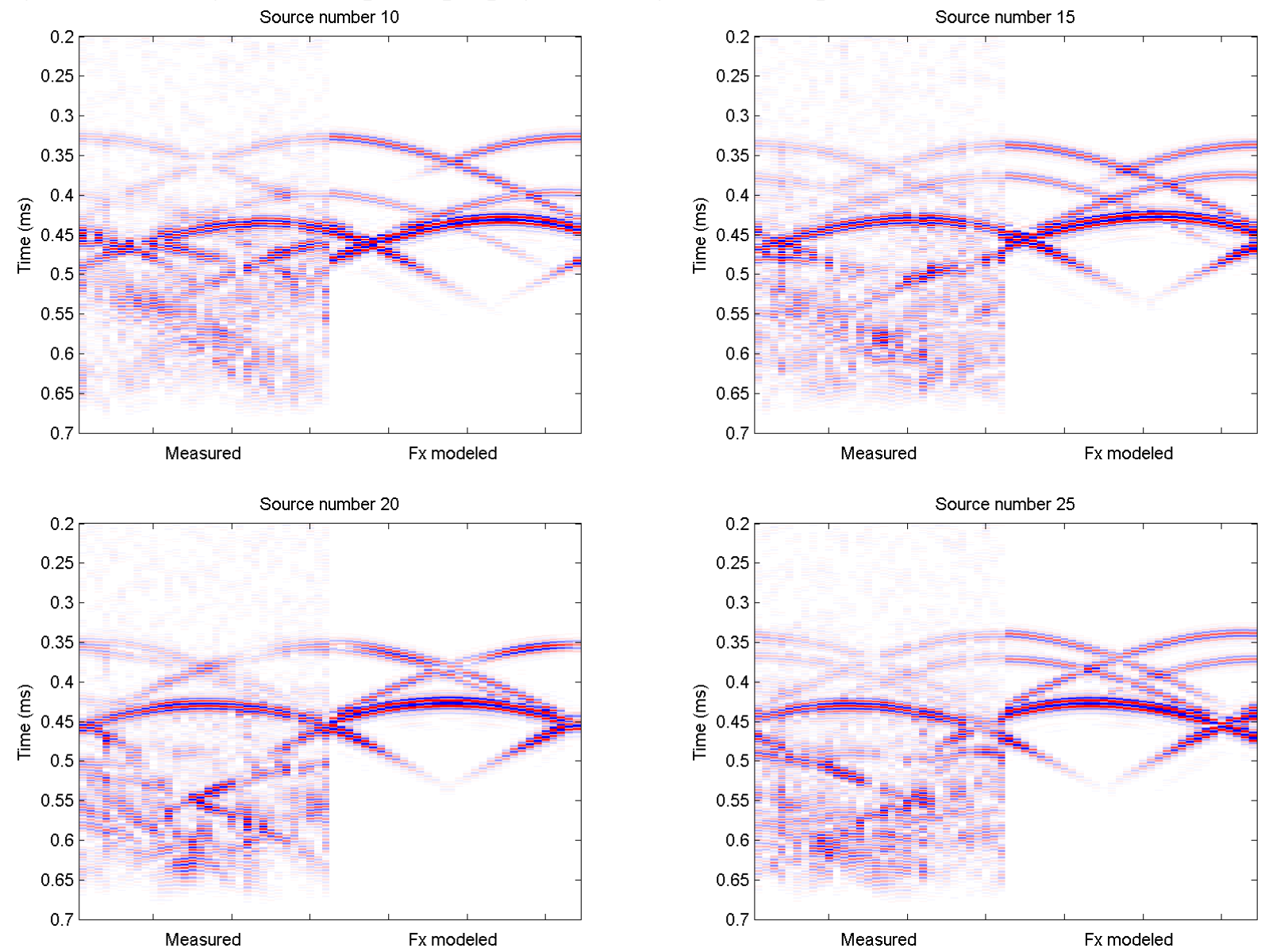

Figure 6 Comparison of the measured and modeled wave fields for four sources (number $5,10,15$ and 20). The left side of each panel shows the measured wave fields at 32 receivers and the right side shows modeled wave fields.

\section{Tomographic inversion}

A tomographic inversion scheme has been developed that uses the recursive wave field extrapolation operators as kernel. This replaces the conventional ray-tracing approach with a more accurate method. The inversion scheme is applied to modeled data.

The inversion scheme uses an iterative approach and adaptive grid refinement at locations where wall thickness loss is detected.

All modeled defects have a depth of $20 \%$ of the nominal wall thickness. We illustrate the inversion using a $90^{\circ}$ bend, with a wall thickness of $8.3 \mathrm{~mm}$.

The inversion results are shown in Figure 7, for two different frequencies $80 \mathrm{kHz}$ and $160 \mathrm{kHz}$. At both frequencies the corrosion spot is recovered, however the result at $160 \mathrm{kHz}$ is clearly better. This is due to that fact that the dispersion effects are much more pronounced. 


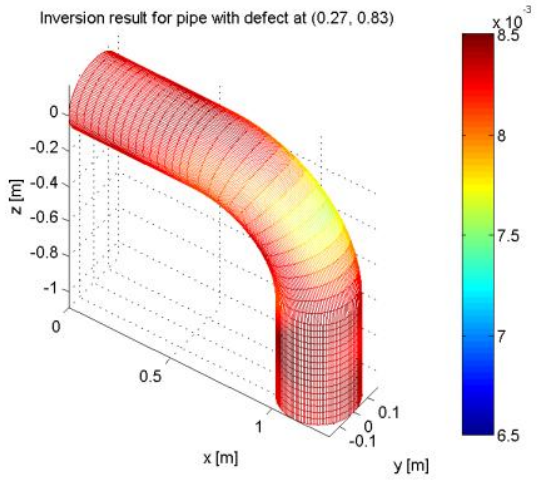

a)

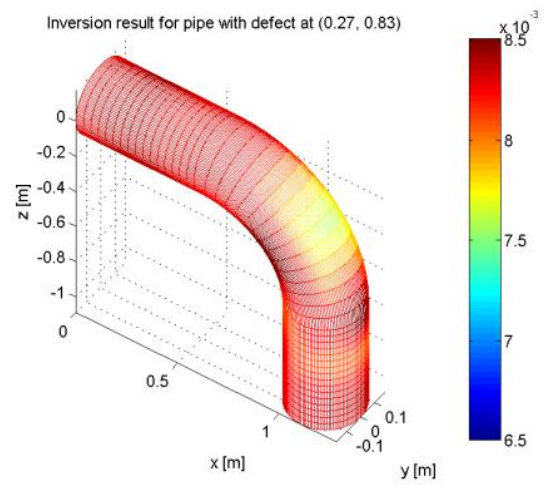

c)

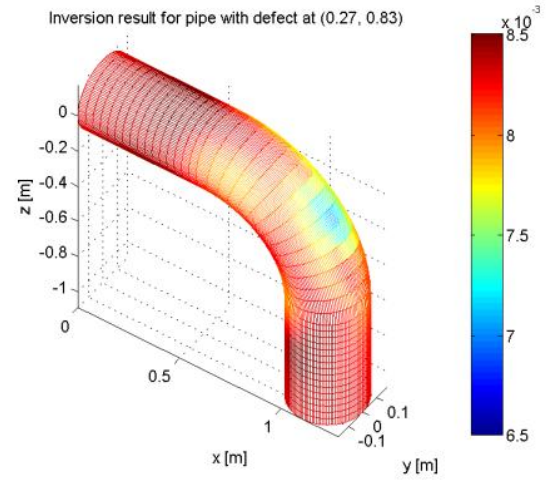

b)

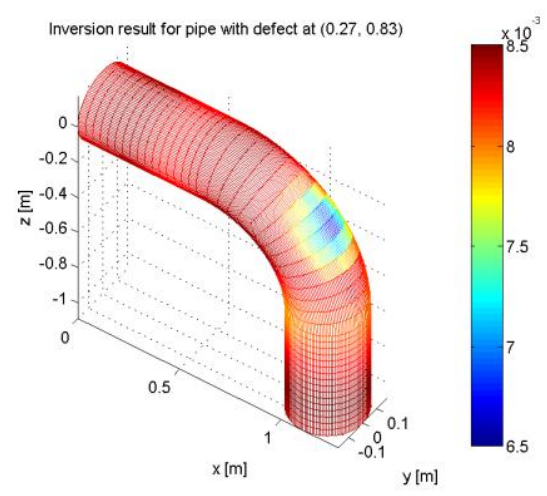

d)

Figure 7 Inversion results, a) $80 \mathrm{kHz}$, initial grid, b) final result of $80 \mathrm{kHz}$ (with adaptive grid refinement, c) $160 \mathrm{kHz}$, initial grid, d) final result of $160 \mathrm{kHz}$

As explained in section 2, guided waves tend to focus on the knee of the bend. This means that many wave paths traverse through this point. Hence it is expected that a defect on the knee can be imaged. A dataset is modeled where the defect is at a different location in the bend, the depth is still $20 \%$ of the wall thickness. The final tomographic inversion result is shown in Figure 8. The results show that also this defect can be reconstructed quite well. 


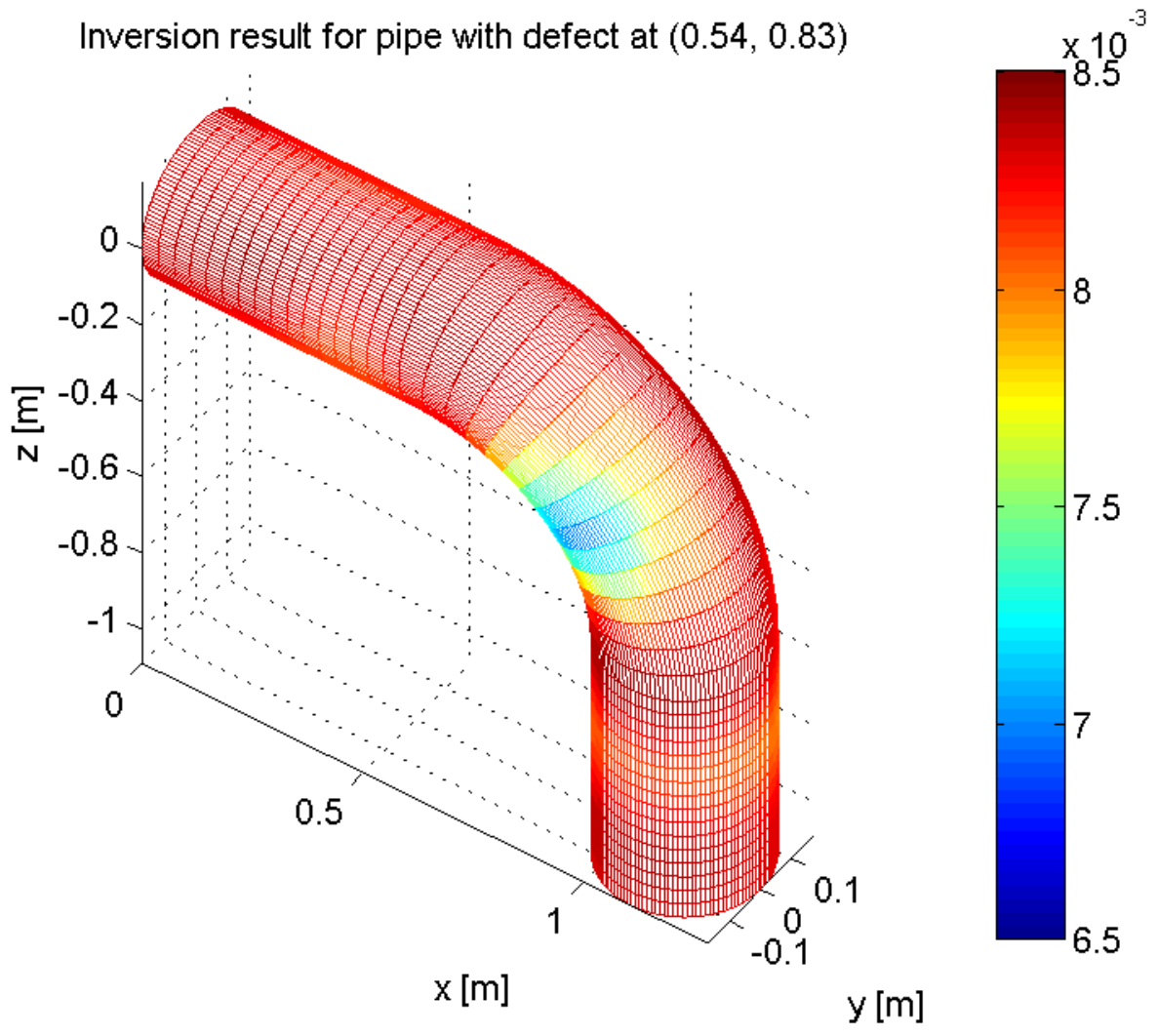

Figure 8 Tomographic image of a corrosion spot with $20 \%$ wall thickness loss.

\section{Concluding remarks}

In this paper the application of travel time tomography on bends was discussed. As was shown, travel time tomography is difficult to apply due to phase shifts of the waves due to geometrical focusing. Therefore a new forward modeling kernel for tomography was developed which is based on wave field extrapolation. By including an optimization routine, short operators can be used, which allow a good balance between accuracy and speed. The model was validated by comparing the results with an experimentally recorded dataset. It showed that the model correctly predicted the properties relevant for travel time tomography: events, travel times and the phase shift. There were some amplitude differences observed due to directivity of the transducers, but this can be easily included in the model.

A tomographic inversion scheme has been developed using the recursive wave field extrapolation operators. The scheme was tested on a $90^{\circ}$ bend, with defect with a depth of $20 \%$ of the wall thickness. The results indicate that the defect can be recovered accurately, impendent of its location. 


\section{References}

1. Arno Volker, Arjan Mast and Joost Bloom, "Experimental results of guided wave travel time tomography", Review of Progress in Quantitative Nondestructive Evaluation, Vol. 29, eds. D. O. Thompson and D. E. Chimenti, pp. 2052-2059, published by AIP (Melville, NY), 2009.

2. Schubert F. et al., 1998, The elastodynamic finite integration technique for waves in cylindrical geometries, J. Acoust. Soc. Am. 104 (5), 2604-2614, 1998.

3. J.W. Thorbecke, Common Focus point Technology, PhD-thesis, Delft University of Technology, 1997. 